\title{
NEUTRON-PROTON SCATTERING
}

\author{
BY H. N. YADAV \\ Physics Dipartmint, Scilinch, Coldirir, Paina
}

(Reccived for publicalion, Fibruary $2.3,19.52)$

ABSTRACT. The angular distribution and the total cross section have been calculated for incident neutrons of cnergy $83 \mathrm{Mev}$ scattered by protons. Interactions considered are of symmetrical type and one with int'raction in the even states only, for Yukawa potential with ranges:

$1.18 \times 10^{-13} \mathrm{~cm}$ for singlet states

$0.80 \times 10^{-13} \mathrm{~cm}$ for the central part in the triplet states

and

I. $60 \times 10^{-13} \mathrm{~cm}$ for the non-central part in the triplet states.

The constants for the singlet putential are taken to fit the proton protun scattering and the low.energy neutron-proton scattering. The constants for the triplet potential are dctermined by the interaction method to fit the binding energy, the electric quadrupole moment and the magnetic moment of the deuteron. The low energy scatterng total cross sections obtained are in agreement with the experimental values. But the higb energy $\left(8_{3} \mathrm{Mev}\right)$ total cross sections are large as usual, viљ., for symmetrical interaction it is $14.6 \times 10^{-26} \mathrm{~cm}^{2}$ and for intcraction in the even states only, it is $12.1 \times 11^{-26} \mathrm{~cm}^{2}$.

\section{INTR()I)【゙やIUN}

The theoretical investigations of high encrgy n-p scattering by severai

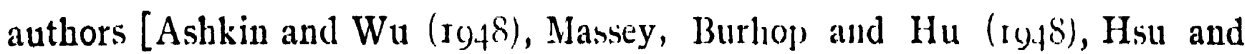
$\mathrm{Hu}$ (1949), Rohrlich and Iïsenstein (Ig/9), Wu and Foley ( $(948)$, Burhop and Yadav (1948, 1949)」 give values for the lotal cross section too large compared with the experimental results. The only investigation so far which gives results in agreement with the experiment is that of Camac and Bethe (1948) for the case of a spherical well potential consisting only of a central force of range $2.0 \times 10^{-13} \mathrm{~cm}$.

In the present calculation, a brief reference of which was made by the author (Yadav 1949), the non-central force has been retained in order to account for the clcctric quadrupole moment of the deuteron. Also, the singlet range has been taken different from the triplet ones, as is suggested by the analysis of the experiment of the scattering of thermal neutrons by the para-and ortho-hydrogen molecules (Sutton 1947). Moreover, for the triplet states the range for the central force has been taken different from that of noncentral forces, as the same range for the central and non-central forces do not seem to be consistent with the requirement of the various experimental data. For, if we consider Yukawa potential of a single range for both the central and non-central forces, then in order to satisfy the scattering of 
thermal neutrons by bydrogen molecules the range should be between $0.75 \times 10^{-13} \mathrm{~cm}$. and $1.06 \times 10^{-13} \mathrm{~cm}$. On the other hand, the electric quadrunole moment and the magnetic moment of the deutcron cannot both be fitted if equal range of value in the above interval be chosen for both the central and non-central forces. This requirement can, however, be fulfilled if a larger range of the non-central force is used. Besides, none of the attempts with cqual range, as already mentioned, has yielded results in agreement with experiments on high encrgy scattcring.

Castillejo and Richardson (1949) have carried out calculations for 83 Mev n-p scattering, with different central and non-ccutral ranges for a spherical well interaction with ranges and constants given by Miss Padfield (1949). The values of the total cross section are again very large. Taking all these facts in view it was considered worthwhile to undertake the calculation with Yukawa potential with range $1.18 \times 10^{-1 s} \mathrm{~cm}$ in the singlet states and $0.8 \times 10^{-13} \mathrm{~cm}$ for the central triplet and $1.6 \times 10^{-13} \mathrm{~cm}$ for the triplet non-central potential. All the constants were calculated to fit the low energy scattering and the properties of the deuteron. However, this new attempt has not been any more successful than the previous attempts with a single range for $83 \mathrm{Mev}$ neutrons scattered by protons.

\section{DETRMINATION OF CONSTANTS ANDI,OW E N E R Y S C $\triangle$ T T E R ING}

(a) Determination of constants in the expression for the interaction:

(i) Triplet slates-The potential assumed is of the form

$$
' V=\frac{\overrightarrow{1^{\prime} \cdot 2}}{3} \frac{\hbar^{2}}{M r_{0}^{2}}\left\{a \frac{e^{-2 r / r_{0}}}{r / r_{0}}+a \gamma S_{12} \frac{e^{-r / r_{0}}}{r / r_{0}}\right\}
$$

which in the case of even states reduces to

$$
{ }^{3} V_{\text {oven }}=-\frac{\hbar^{2}}{M r_{0}^{2}}\left\{a \frac{e^{-2 r / r_{0}}}{r / r_{0}}+a \gamma S_{12} \frac{e^{-r / r_{0}}}{r / r_{0}}\right.
$$

where $r_{0}=1.6 \times 10^{-13} \mathrm{~cm}$ is the range for the non-central force and $r_{0} / 2=$ $.8 \times 10^{-11} \mathrm{~cm}$ is that for the central force. Other symbols have their usual meaning.

Assuming the binding energy of deuteron, the wave equations for the ground state of deuteron are solved by the interaction method. The constants $a$ and $a \gamma$ are determined by making use of the expression and the experimental value of the electric quadrupole moment of the deuteron. The values obtained are as follows :

$$
a=2.662 \text {, } a \gamma=1.835 \text {, and } D \%=3.78 \text {, }
$$

satiofying the requirement of the magnetic moment of the detteron. 
(ii) Singlet slates:-The potential in this case is of the form

$$
{ }^{2} V=-\frac{\hbar^{2}}{M r_{s}^{2}} \overrightarrow{\tau_{1}} \cdot \overrightarrow{\tau_{2}} g \frac{e-\tau / r_{0}}{r / r_{s}}
$$

which for the even states becomes

$$
{ }^{2} V_{\text {evon }}=-\frac{\hbar^{2}}{M r_{s}^{2}} g \frac{e^{-r r_{0}}}{i / r_{a}}
$$

The range $r_{s}=1.18 \times 10^{-13} \mathrm{~cm}$. and the potential constant $g=\mathrm{r} .575$, Thes? values were calculated by Hsu who determined thein to give correctly the low-energy n-p scattering.

(b) The scattering of low-energy neutroins by protons

In calculating the total cross section for the scattering of low encrgy neutrons by protons the following approximate expressions ( $W_{11}$ and Foley 1949) have been used

$$
k_{1} \cot \delta_{1}-k_{2} \cot \delta_{2}=\left(k_{2}{ }^{2}-k_{1}{ }^{2}\right) \int_{0}^{\infty}\left(v_{0}{ }^{2}-u_{0}{ }^{2}\right) d r .
$$

for the singlet scattering, where $k_{1}=\frac{M E_{1}}{\hbar^{2}}$ and $\delta_{1}$ is the phase shift of the wave for incident neutron of energy $E_{1} ; u_{0} / r$ is the radial part of the wave function for zero energy and tends asymptotically to $v_{0} / r$. For the triplet scattering,

$$
k \cot \delta=-\alpha+\left(\alpha^{2}+k^{2}\right) \int_{0}^{\infty}\left\{v_{\alpha}^{2}-\left(u^{2}+v^{2}\right)\right\} d r .
$$

where $\alpha^{2}=\frac{M\left|E_{0}\right|}{\hbar^{2}}, F_{0}$ being the binding energy of the deuteron and $\frac{u}{r}, \frac{w}{r}$ are respectively the radial parts of the $S$ and $D$ wave of the ground state of the deuteron and normalized such that $u$ tends asymptotically to $v_{a}=e^{-a r}$. Also $k^{2}=\frac{M E}{\hbar^{2}}$ and $\delta$ is the phase shift of the wave for incident neutron of energy $E$.

The total cross sections calculated by using ( $I$ ) and (2) are in good agreement with those calculated by the exact method. This has been verified for the case of a spherical well interaction up to $6 \mathrm{Mev}$. We expect that in the case of the Yukawa potential it will give results in agreement with exact calculations up to at least $3 \mathrm{Mev}$. The total cross sections obtained are given in the Table I. As seen from figure $\mathrm{x}$. they are in good agreement with experimental values obtained by various authors. 


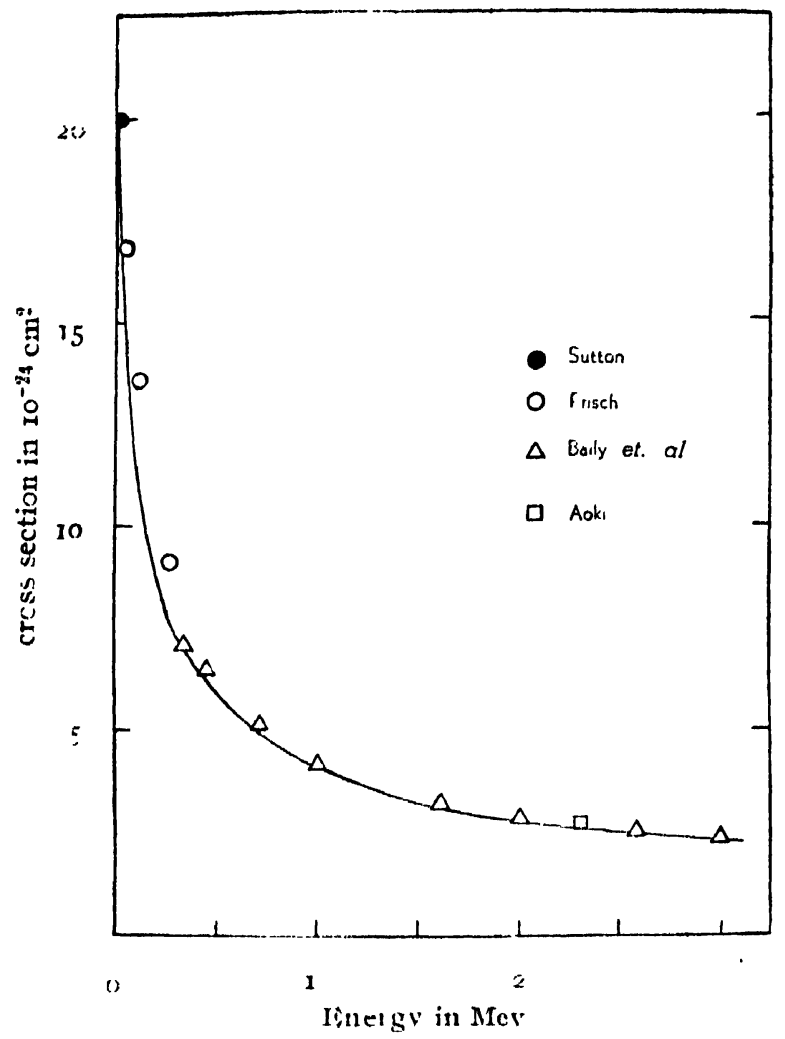

Fig. I

TABLE: I

\begin{tabular}{c|c}
\hline Tinergy of incident neutron in Mev. & T.stal cross section in $10^{-21} \mathrm{~cm}^{2}$. \\
\hline 0 & 1959 \\
.1 & 12.35 \\
.5 & 5.94 \\
1.0 & 4.11 \\
1.5 & 329 \\
20 & 280 \\
2.5 & 2.46 \\
30 & 220 \\
\hline
\end{tabular}

3. SCATTERING OF 83 MEV NEUTRONS BY PROTONS.

(i) Triplet states:-The phases for the coupled states ${ }^{3} S_{1}$ and ${ }^{3} D_{1}$ and for the uncoupled states ${ }^{3} P_{0},{ }^{3} P_{1}$ and ${ }^{3} D_{2}$ were calculated from the solutions of the radial wave equations obtained exactly by numerical integration. All higher phases, being very small, are taken account of by Born's approximation. The procedure adopted is briefly outlined below. 
For an incident plane wave $e^{1 /}: X_{1}$ the outgoing scattered spher ical wave can be written as (Hu and Massey I() 49 )

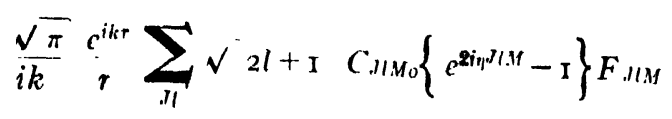

where the notations are the same as in the paiper referred to above. From (3) the scattering amplitude can at once be written down as

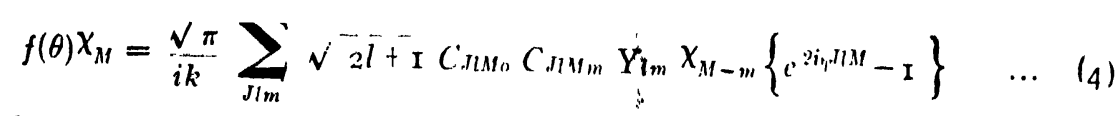

If we define the matrix element for the scittering amplitude by

$$
\left.f(\theta)_{M}, 1\right)=X_{M} f(\theta) X_{M}
$$

i.e. the probability amplitude of scattering of the incident wave with spin component $M$ into a scattered wave with spin component $M^{\prime}$ then from (4)

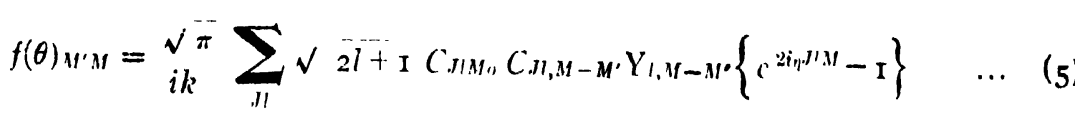

Hence the ${ }^{3} \sigma(\theta)$ is given by

$$
{ }^{\prime} \sigma(\theta)=! \sum_{M^{\prime}} \sum_{M}\left|f(\theta)_{M^{\prime}, M}\right|^{2}
$$

where the summations indicate averaging over the initial spin states and summing over the final spin states.

In the case of Born's approximation (Ashkin and Wu ro48; Burhop) and Yadav I94\$, I949) for the symmetrical interaction

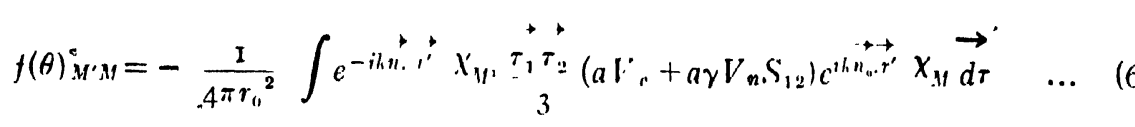

where $V_{c}=\frac{e^{-2 r^{\prime} / r_{0}}}{r^{\prime} / r_{0}}$, the central qotential

and $\quad V_{n}=\frac{e^{-r \%_{0}}}{v^{\prime / r}}$, the won contral potential

The expression (6) can be integrated out explicitly in the form

$$
\begin{aligned}
& f(\theta)_{M^{\prime} M}=\frac{1}{3} a^{\hat{S}_{M^{\prime}} M}\left[2 F\left(\pi-\theta_{1}+F(\theta)\right]+\frac{1}{3} a \gamma\left[2 \Gamma_{M^{\prime} M}(\pi-\theta, \pi+\varphi) c(\pi-\theta)\right.\right. \\
& \left.+\Gamma_{M^{\prime} M}(\theta, p)_{C}(\theta)\right] \ldots \quad(7)
\end{aligned}
$$

The various terms occurring here are explained and worked out in the papers referred to above. $(\theta, \varphi)$ are the angular coordinates of $\vec{n}$ referred to $\overrightarrow{n_{1}}$.

In the case of interactions in the even states only, as suggested by Serber, $f(\theta)_{M^{\prime} M}$ are obtained from $(5)$ if the terms associated with odd $l^{\prime}$ 's are omitted. And it can easily be shown that the Born amplitudes in this case are given by

$4-3802 \mathrm{P}-7$ 


$$
\begin{aligned}
f(\theta)_{M^{\prime} M}=\frac{1}{2} a \delta_{M^{\prime} M}[F(\pi-\theta)+F(\theta)]+\frac{1}{2} a \gamma\left[\Gamma_{M^{\prime} M}(\pi-\theta, \pi+\varphi) C(\pi-\theta)\right. & \\
& \left.+\Gamma_{M^{\prime} M}(\theta, \varphi) C(\theta)\right] \quad \ldots \quad(7 a)
\end{aligned}
$$

T'o take account, by Born's approximation, of the contribution from states of higher angular momenta, for which phases were not calculated by exact inethod, the foilowing prosedure was adopted. The amplitudes were calculated by Born's approximation using expressions (7) and (7a) which give contribution from all $l$ values. To this is added the expression

$$
f(\theta)_{M^{\prime} M} \text { (exact) }-f(\theta)_{M^{\prime} M}(\text { Born })
$$

for the states ${ }^{3} S_{1},{ }^{3} D_{1},{ }^{3} P_{0},{ }^{3} P_{2}$ and ${ }^{3} D_{3}$. This has the effect of using the exact phases in place of Born ones for the states in question while retaining all the Born phases for higher states. In order to evaluate (8) the expression (6) for $f(\theta)_{M^{\prime} M}$ (Born) is to be cast in the form (5). The incident wave $c^{i k z} X_{M}$ can be expanded in the form

$$
e^{i k z} X_{H}=\sum_{., 1} \sqrt{4 \pi(2 l+I)} i^{l} C_{. l l M_{0}} \frac{g_{l}(k r)}{k r} F_{J I M}(\theta . \varphi)
$$

Following Mott and Massey (1950), the Born phases can easily be deduced in the form

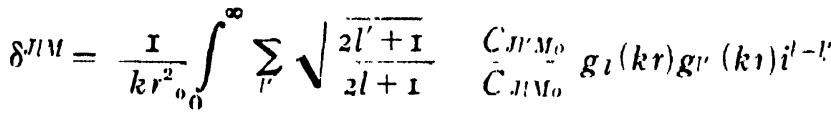

$$
\begin{aligned}
& \times\left(a V_{c} \delta_{1}{ }^{\prime}+a \gamma V_{n}<l\left|S_{12}\right| l^{\prime}>\right) d r \quad \ldots \quad \text { (10) }
\end{aligned}
$$

where

$$
\left.<l\left|S_{12}\right| l^{\prime}\right\rangle=\left\langle F^{*}, M_{M}\left|S_{12}\right| F_{, I^{\prime} M}\right\rangle
$$

and has the following values:

$$
\begin{aligned}
& \left\langle J-\mathrm{I} \cdot\left|S_{12}\right| J-I\right\rangle=-\frac{2(J-I)}{2 J+I} \\
& \left\langle J\left|S_{12}\right| J\right\rangle=+2 \\
& \left\langle J+I\left|S_{12}\right| J+I\right\rangle=-\frac{2(J+1)}{2 J+I} \\
& \left\langle J F_{\mathrm{I}}\left|S_{12}\right| J \pm \mathrm{I}\right\rangle=\frac{6 \sqrt{J(J+I)}}{2 J+1}
\end{aligned}
$$

all other elements vanishing.

Also

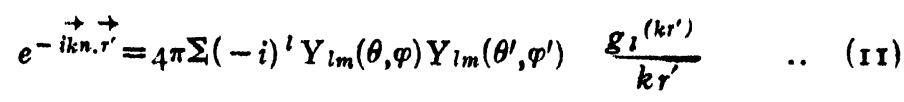

where $\theta, \varphi$ are the co-latitude and azimuth angles of $\vec{n}$ referred to $\vec{n}_{0}$ and $\theta^{\prime}, \varphi^{\prime}$ are those for $\vec{r}^{+}$referred to $\overrightarrow{n_{0}}$. Substituting the expansions $(g)$ and (II) in (6) and integrating over the spin and the angular coordinates we get 


$$
\begin{aligned}
f(\theta)_{M^{\prime} M}(\text { Born })= & \frac{I}{r_{0}^{2}} \cdot \int \sum_{J l^{\prime}}(i)^{l^{\prime}-l} C_{J I M, M-M^{\prime}} C_{I l^{\prime}, M_{0}} Y_{l, M-M^{\prime}} \\
& \times \sqrt{4 \pi\left(2 l^{\prime}+\bar{I}\right)}\left(a V_{c} \delta_{l l^{\prime}}+a \gamma V_{n}<l\left|S_{12}\right| l^{\prime}>\right) \frac{g_{l}^{\left(k r^{\prime}\right)} g_{k^{2}} l^{\left.l r^{\prime}\right)}}{k^{\prime}} d_{1}
\end{aligned}
$$

which according to (10) gives

$f\left(\theta_{M^{\prime} \cdot M}(\right.$ Born $)=\frac{v^{\prime} \bar{\pi}}{i k} \sum_{\pi} \sqrt{(2 \bar{l}+\mathrm{I})} C_{J I M, M-M^{\prime}} C_{J I M}, \mathbf{Y}_{1, M-M^{\prime}}(\theta, p)\left(2 i \delta^{J l M}\right) \quad \ldots \quad$ (1 2$)$

(we use $\delta^{J l M}$ for Born's phases and $\eta^{J l u}$ for exact oncs).

Hence

$$
\begin{aligned}
& f_{M^{\prime} M}(\theta)(\text { exact })-f_{M^{\prime} M}(\theta)(\text { Born }) \\
& \left.=\frac{\sqrt{\pi}}{i k} \sum_{J l} \sqrt{(2 l+I)} C_{J M, M-M^{\prime}} C_{J I M} Y_{l, M-M} \cdot e^{2+\eta n J l M}-I-2 i \delta^{J M}\right\} \quad \ldots \quad\left(1_{3}\right)
\end{aligned}
$$

(ii) Singlet states : In the singlet states, there being only one spin function, the amplitude of scattering for Born's approximation is given by

$$
f(\theta)=g[2 F(\pi-\theta)-F(\theta)] \text { for symmetrical interactions }
$$

and $f(\theta)=\frac{1}{2} g[F(\pi-\theta)+F(\theta)]$ for interaction in the event states only.

Also the expression (8) for the singlet scattering can easily be shown to be

$$
f(\theta)\left(\text { exact } ;-f(\theta) \text { (Born) }=\frac{I}{2 i k} \underset{l}{\mathrm{~S}}(2 l+1) P_{l}^{\prime}(\cos \theta)\left\{e^{2 i l^{l}-1}-2 i \delta^{l}\right\} \quad \ldots \quad \text { (I }_{1}\right)
$$

'The factor $\left(e^{2 i \eta}-1-2 i \delta\right)$ both in $\left(x_{3}\right)$ and $(14)$ is negligible if $"$ is small. This is approximately true for the states for which exact phaser are not calculated. Thus the Born's approximation for these states wiil give almost the same contribution as the exact calculation.

\begin{tabular}{|c|c|c|c|c|}
\hline & \multicolumn{2}{|c|}{${ }^{3} S_{1}$} & \multicolumn{2}{|c|}{${ }^{3} D_{1}$} \\
\hline & $M= \pm \mathbf{I}$ & $M=0$ & $M= \pm I$ & $M=\mathrm{c}$ \\
\hline Fxact $\left(e^{2 i n}-x\right)$ & $1799+.819 t$ & $-1.364+.501 t$ & $-.413-197 i$ & $+.021-515 i$ \\
\hline Born 218 & $+.777^{i}$ & $+1.615 i$ & $-.952 i$ & $-.114 i$ \\
\hline
\end{tabular}

\section{R U S U L T S}

The phases obtained are given in 'I'able II and the angular distribution in Table III. Figure 2 represents the angular distribution graphically.

TABLK II

(a) Iriplet coupled phases 
(b) Triplet uncoupled phases

\begin{tabular}{l|c|c|c|c}
\hline & ${ }^{3} P_{0}$ & $3 P_{1}$ & ${ }^{3} D_{2}$ & \\
\hline$\eta$ (Hixact) & +30 & -.191 & +.266 & \\
$\delta$ (Bort) & +.305 & -.207 & +.250 & \\
\hline
\end{tabular}

(c) Singlet phases

(These phases are taken from Hsu's calculations)

\begin{tabular}{l|c|c|c}
\hline & $S$ & $P$ & $D$ \\
\hline Exact $\eta$ & +.571 & -.565 & +105 \\
IBorn $\delta$ & +.565 & -.741 & +.099 \\
\hline
\end{tabular}

\section{TABLE III}

(a) Angular distribution (in $10^{-26} \mathrm{~cm}^{2}$ )

\begin{tabular}{c|c|c|c|c|c|c|c|c|c}
\hline$\theta 0$ & 0 & 15 & 30 & 60 & 90 & 120 & 150 & 165 & 180 \\
\hline Sym. $\sigma(\theta)$ & 1.488 & 1.430 & 1.300 & .968 & .806 & 1058 & 2081 & 2647 & 2.971 \\
Serber $\sigma(\theta)$ & 1303 & 1322 & 1268 & .887 & 7.33 & .887 & 1268 & 1.302 & 1.303 \\
\hline
\end{tabular}

(b) Total cross sections $\sigma$ and ratio $\begin{aligned} & r\left(180^{\circ}\right) \\ & r\left(90^{\circ}\right)\end{aligned}$

$\begin{array}{lcc}\text { Interaction } & \frac{\sigma\left(180^{\circ}\right)}{\sigma\left(90^{\circ}\right)} \\ \text { Symmetrical } & 14.62 \times 10^{-26} \mathrm{cn1}^{2} & 3.68 \\ \text { Serber } & 1207 \times 10^{-26} \mathrm{cn}^{2} & 1.78\end{array}$

As the Serber interaction gives a symmetrical scattering cross section about $\theta=90^{\circ}$ in the centre of mass system it does not seem to be in agreement with the experimental results, though it reduces the total cross section to some extent. 


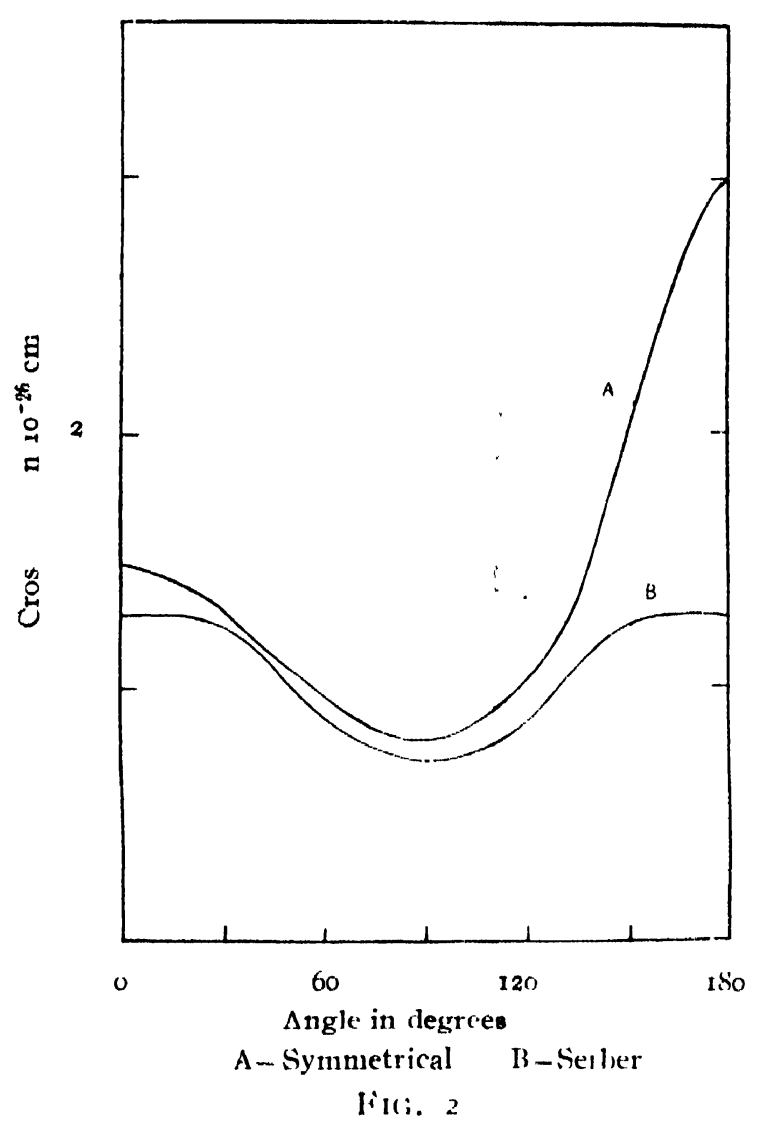

'Thus it hardiy requires to be said that the Yukawa potcntial with different ranges, as attempted in the present calculations, does not give any better result for the high energy scattering than is obtainable with a single range of interactions.

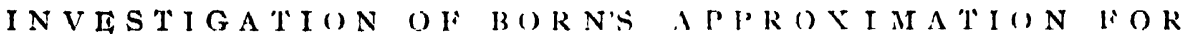
SCATTHRING IN THIS TRIPI,IT STA IIS

It will not be out of place to record here the striking disagreement between the results obtained by using Born's approximation and the exact method for scattering in the triplet states.

Figure 3 shows a comparison of the triplet differential cross sections calculated by the exact method and by Born's approximation. While the angular distribution differs widely in the two cases, the cross section in the triplet states obtained hy Born's approximation is only $8.1 .4 \times 10^{-26} \mathrm{~cm}^{2}$ whereas, the exact calculation gives $\mathrm{I} 4.96 \times 10^{-26} \mathrm{~cm}^{2}$. The reason for the low value for this cross section is the shortness of the range. If the range is taken to be zero then the cross section for all forms of potentials (splerical well, exponential well, Yukawa potential and (Gaussian potential) will be zero if Born's approximation is used, while the exact mathod gives a non- 




vanishing finite value for the cross section. J'hus it scems that unless the range is sufficiently large, Born's approximation will always yield too low a value.

\section{$\triangle C \subset K N O W L E D G M E N S$}

The author wishcs to express his indebtedness to Prof. H. S. W. Massey, F R.S. and Dr. E. H. S. Burhop for their keen interest in the calculation. Thanks are also due to Dr. T. M. Hu aud Dr. K. N. Hsu for allowing the author to use their results of calculation for the singlet states and for many valuable discussions.

\section{R F F F R E C E S}

Ashkin. J., and Wu, T. Y., 1948. Phys. Rev., 73, 973.

Burhup, E. H. S., and Yadav HI. N.. 1948, Nature, 168. 738,

Camac," M.. and Bëthe, H. I949, Proc. Roy Soc., I 197, 505

Castillejo, L., Rechardson, H. T. 1949, Phys. Rev., 76. 1732.

Hsa, K. N. and Hu T. M. 1949, Phys. Riv., 78 987

Hn, T. M. and Mrassey, H S. W., 19.19, Proc Roy. Soc., I 196, 135.

Massey, II. S. W . Burhop, F. H. S. and Hu, T. M., 1948, Phys. Rov., 73, 2403.

l'adfield, Mi ss D. G , 1949, Naturc., 163, 22.

Rohrlich, F., and râisenstein, J, 1949, Phys Rev., 76, 705

Sutton, R. B., et al, 1947, Phys. Rcv., 79, 1147

Wu, T Y. and Foley, H. M., 1949, Phys. Rev., 76, 1681.

Yadav, H. N.. 1949, Phys. Rev., 76, 1720. 